\title{
Maia Mapper: High Definition XRF Imaging of Geological Samples at Intermediate Spatial Scales
}

Chris Ryan $^{1 *}$, Robin Kirkham ${ }^{1}$, David Parry ${ }^{1}$, Gareth Moorhead ${ }^{1}$, Murray Jensen ${ }^{1}$, Steve Hogan ${ }^{1}$, Andrew Faulks ${ }^{1}$, Paul Dunn ${ }^{1}$, Roshan Dodanwela ${ }^{1}$, Louise Fisher ${ }^{1}$, Mark Pearce ${ }^{1}$, Pete Siddons ${ }^{2}$, Anthony Kuczewski ${ }^{2}$, Ulf Lundström ${ }^{3}$, Alexia Trolliet ${ }^{3}$ and Ning $\mathrm{Gao}^{4}$

1 CSIRO Mineral Resources, Clayton VIC 3168, Australia

2 Brookhaven National Laboratory, Upton NY 11973, USA

3 Excillum AB, 16440 Kista, Sweden

4 XOS, East Greenbush, USA

* Corresponding author, chris.ryan@csiro.au

Maia Mapper targets high definition imaging of element distribution in geological samples, such as drill core and large polished rock slabs, at spatial scales between the traditional realms of X-ray microanalysis and imaging, at the (sub)micron to mm scale, and the macroscale, typically sampled in the geological environment by bulk sampling at the meter scale. It uses focused X-rays from a laboratory micro-focus source and builds on the success of the Maia detector array in geology [1] to achieve imaging of up to $\sim 80 \mathrm{M}$ pixels over a $500 \times 150 \mathrm{~mm}^{2}$ sample area, as part of the analytical workflow of the Advanced Resource Characterization Facility, which spans spatial scales from ore deposit to atomic scales.

Maia Mapper combines (see Fig. 1) (i) the Maia detector array and fluorescence imaging system [2,3], with its large solid-angle $(1.3 \mathrm{sr})$ and high count-rate capacity $(>10 \mathrm{M} / \mathrm{s})$, event-mode operation, millisecond pixel transit times in fly-scan mode and real-time spectral deconvolution and imaging [4], (ii) the high brightness MetalJet D2 liquid metal jet micro-focus X-ray source from Excillum (using high In I2 alloy) [5], (iii) an efficient XOS polycapillary lens with a flux gain $\sim 15,900$ at $21 \mathrm{keV}$ into a 32 $\mu \mathrm{m}$ focus and an acceptance at the source of $\sim 43 \mu \mathrm{m}$ (at $24 \mathrm{keV}$ ), (iv) a sample scanning stage engineered for standard drill-core sections, and (v) a shielded enclosure providing radiation and motion safety [6].
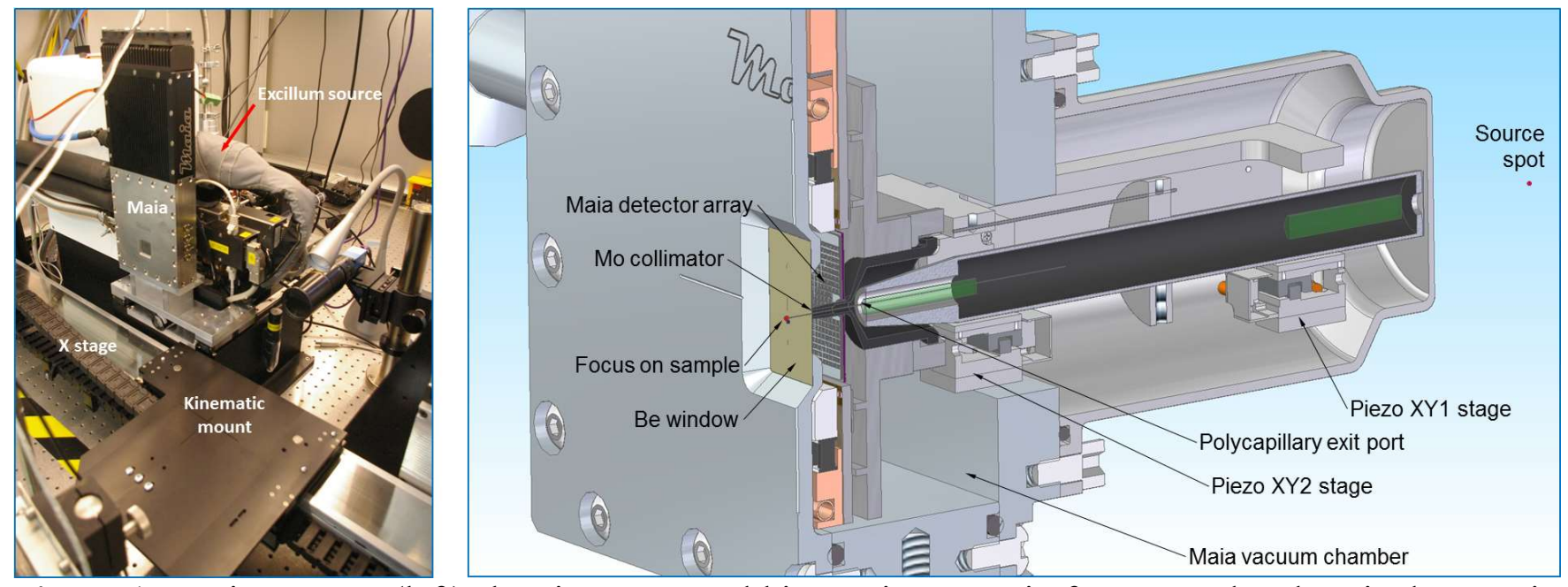

Figure 1: Maia Mapper (left) showing stage and kinematic mount in fore-ground and Maia detector in front of Excillum source, and (right) sectional view of Maia showing the in-vacuum $100 \mathrm{~mm}$-long polycapillary lens on its 4-axis piezo adjuster that aligns it with the source spot and output collimator [6]. 
Areas to be scanned and sample metadata are added to a scan list and stored in a key-value store. Selected scans in the list are run in an automated sequence with Maia event data and metadata logged to cloud storage. DA images, detector spectra and a count-rate map are displayed in real-time using a graphical client.

Count-rates up to $\sim 3 \mathrm{M} / \mathrm{s}$ are observed on drill core samples, with detection from 2 to beyond $32 \mathrm{keV}$ (Ba $\mathrm{K}$ ) and low dead-time per pixel per detector of up to $\sim 1.5 \%$. Typical transit time per $30 \mu \mathrm{m}$ pixel is $2-12$ ms with a distribution reflecting speed variations in the Galil stage controller. The images are normalized to pixel transit time and show no evidence for uncorrected stage speed variations or source intensity fluctuations, which is consistent with accurate time per pixel determination (20 ns precision) and tight Excillum emission current regulation [6]. Automated scans are executed in sequence with display of deconvoluted element component images accumulated in real-time in the Maia detector using the Dynamic Analysis (DA) method [7] of the GeoPIXE software [8].

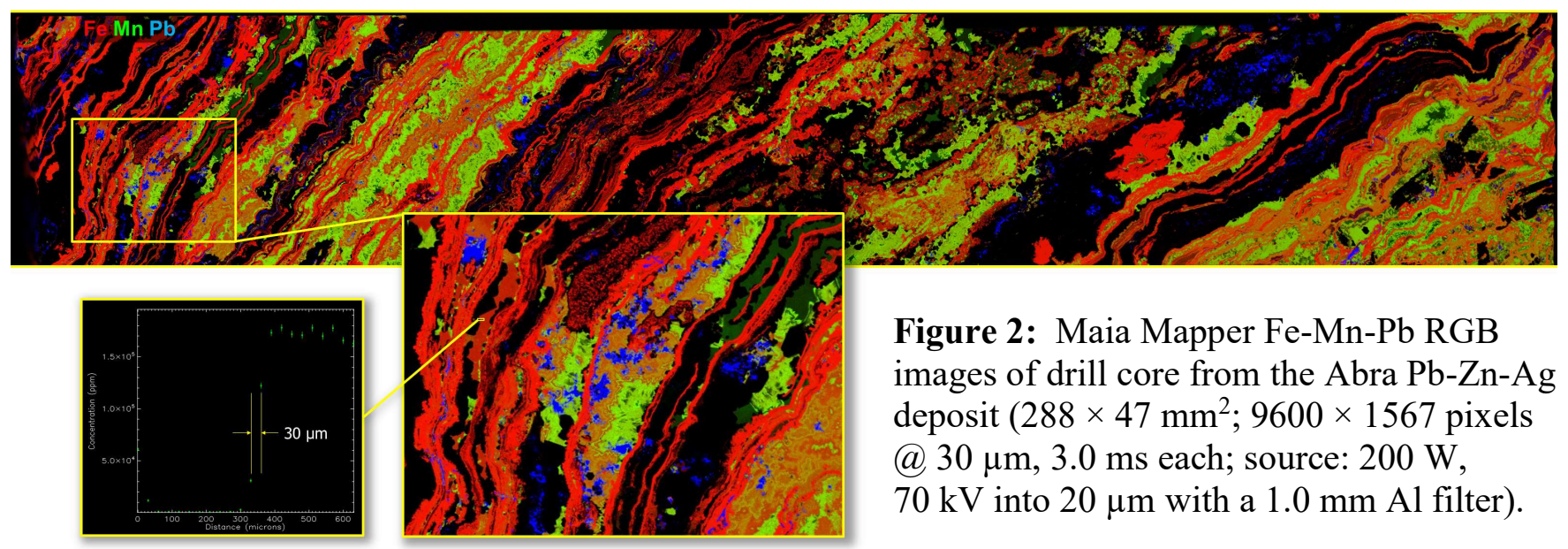

Applied to drill core and rock slabs in mineral exploration or ore body delineation campaigns, the Maia Mapper yields element images of up to $80 \mathrm{M}$ pixels with a spatial resolution of $\sim 32 \mu \mathrm{m}$, as observed on sharp euhedral grain boundaries (e.g. Fig. 2). Despite this large beam and pixel size, the system is sensitive to rare, microscopic precious metal particles with detection limits for single gold particles down to $3 \mu \mathrm{m}$ in diameter [6]. Hence, it is possible with Maia Mapper to detect single gold particles down to $3 \mu \mathrm{m}$ in diameter in an area of $\sim 100 \mathrm{~cm}^{2}$ at a rate of $\sim 10$ minutes per $\mathrm{cm}^{2}$ [9].

[1] LA Fisher et al., Mineral. Deposita 50 (2015) 665.

[2] DP Siddons et al., Journal of Physics: Conference Series 499 (2014) 012001.

[3] R Kirkham et al., AIP Conference series 1234 (2010) 240.

[4] CG Ryan et al., Journal of Physics: Conference Series 499 (2014) 012002.

[5] DH Larsson et al., Rev. Sci. Instrum. 82 (2011) 123701-1-5.

[6] CG Ryan et al., J. Instrumentation 13 (2018) C03020.

[7] CG Ryan, Int. J. Imaging Systems and Technology 11 (2000) 219.

[8] CG Ryan et al., AIP Conference Proc. 1221 (2010) 9.

[9] Maia Mapper development and the Advanced Resource Characterisation Facility (ARCF) are supported by the Science and Industry Endowment Fund. 